\title{
Mindfulness During Pregnancy and Parental Stress in Mothers Raising Toddlers
}

\author{
Myrthe G. B. M. Boekhorst ${ }^{1,2} \cdot$ Eva S. Potharst ${ }^{3,4} \cdot$ Annemerle Beerthuizen $^{2} \cdot$ Lianne P. Hulsbosch $^{1} \cdot$ Veerle Bergink $^{5}$. \\ Victor J. M. Pop ${ }^{1} \cdot$ Ivan Nyklíček ${ }^{1}$
}

Published online: 6 May 2020

(C) The Author(s) 2020

\begin{abstract}
Objectives Parental stress has been associated with adverse child outcomes and maternal functioning. Yet, maternal factors during pregnancy that can potentially protect against later parental stress, such as mindfulness, have not been studied. Therefore, we aimed to address the possible associations between prenatal mindfulness and levels of parental stress in mothers raising toddlers.

Methods Women in the current study $(n=165)$ were prospectively followed from pregnancy until 3 years after childbirth, as part of a longitudinal population based cohort (HAPPY study). At 22 weeks of pregnancy, women completed the Three Facet Mindfulness Questionnaire-Short Form (TFMQ-SF) to assess facets of mindfulness (acting with awareness, non-judging, and non-reacting). Women completed the Parenting Stress Questionnaire (PSQ) 3 years after childbirth, reporting on three components of parental stress: (1) parent-child relationship problems, (2) parenting problems, and (3) role restriction.

Results Results of multiple linear regression analyses showed that the acting with awareness facet of mindfulness during pregnancy was a significant predictor of fewer parent-child relationship problems and parenting problems, even when adjusted for prenatal and maternal distress and child behavior problems. Higher levels of non-reacting during pregnancy were also significantly associated with fewer parenting problems in mothers raising toddlers.

Conclusions The current study emphasizes that mindfulness during pregnancy may be a protective factor for later parental stress. More research is needed to confirm these findings and to evaluate the possible benefit of a mindfulness intervention training during pregnancy on parenting and child outcomes.
\end{abstract}

Keywords Facets of mindfulness $\cdot$ Parental stress $\cdot$ Pregnancy $\cdot$ Parenting $\cdot$ Acting with awareness

Raising a young child is accompanied by new changes and demands, and can be described as an overwhelming

Myrthe G. B. M. Boekhorst

m.g.b.m.boekhorst@uvt.nl

1 Department of Medical and Clinical Psychology, Tilburg University, Warandelaan 2, 5037 AB, PO Box 90153, 5000 Le

Tilburg, The Netherlands

2 Department of Psychiatry, Erasmus MC, University Medical Centre Rotterdam, Rotterdam, The Netherlands

3 UvA minds, academic outpatient (child and adolescent) treatment center of the University of Amsterdam, Amsterdam, The Netherlands

4 Research Institute of Child Development and Education, University of Amsterdam, Amsterdam, The Netherlands

5 Departments of Psychiatry and Obstetrics, Icahn School of Medicine at Mount Sinai, New York, USA experience by parents (Nyström and Öhrling 2003), and can include experiences of parental stress (Hildingsson and Thomas 2014). Parental stress can be defined as the "aversive psychological reaction to the demands of being a parent" (Deater-Deckard 1998, p. 315). Parental stress consists of various underlying components, such as parent-child relationship problems, parenting problems (competence and skills), and role restriction (Vermulst et al. 2012). According to Abidin (1992), parental stress is influenced by parent and child characteristics, partner relationships, and environmental factors. Parental stress has been found to negatively influence children's social, emotional, and behavioral development (Anthony et al. 2005; Barroso et al. 2018; Crnic et al. 2005; Haapsamo et al. 2013). A good parent-child relationship can protect against child behavior problems (Matthijssen et al. 1998). In turn, child temperament is also a risk factor for parental stress (Saisto et al. 2008), suggesting a transactional relationship between child behavior and parental stress (Neece 
et al. 2012). Not only can parental stress negatively impact child outcomes, it has also been associated with maternal functioning and health, such as increased symptoms of depression (Leigh and Milgrom 2008; Kerstis et al. 2016) and parental stress later in parenthood (Östberg et al. 2007). Thus, different factors seem to contribute to parental stress. In turn, parental stress is a risk factor for both poor child and parentrelated outcomes.

The risk factors for parental stress do not derive after childbirth, but may already exist previously, such as during pregnancy. This is not surprising, given the fact that already during pregnancy, women are making the transition to parenthood (Glover and Capron 2017), and healthy adjustment to this transition is important for developing parental self-efficacy (Mihelic et al. 2016). Also, maternal bonding to the baby develops during pregnancy and is predictive of bonding in the first year after the birth of the baby (Cuijlits et al. 2019). Emotional investment in the pregnancy and the unborn baby results in the development of a new intrapsychic organization, the Motherhood Constellation (Stern 2018). This means that the mothers develop a healthy preoccupation with the wellbeing and protection of the (unborn) baby and by their ability to take care well of their baby. This implies that some feelings of worry may be part of a normal process of adjustment to the transition to parenthood. However, prevalence of both depression and anxiety disorders is heightened during pregnancy (Dennis et al. 2017; Woody et al. 2017), and symptoms of prenatal (during pregnancy) depression and anxiety have been linked to parental stress up to 6 months postpartum. Misri et al. (2010) found that both symptoms of anxiety and depression during pregnancy were significantly associated with parental stress 3 and 6 months postpartum, which was not influenced by antidepressant intake during pregnancy. A longitudinal study by Huizink et al. (2017) showed that symptoms of anxiety during pregnancy, as well as symptoms of trait anxiety, were associated with parental stress 3 months postpartum, but depressive symptoms during pregnancy were not. Furthermore, more anxious attachment styles (e.g., worries about relationships) and more avoidant attachment styles (e.g., distancing oneself) in close social relationships, as reported by mothers during pregnancy, have been associated with higher levels of parental stress 12 months postpartum (Moe et al. 2018).

Even though these studies have shown that risk factors for parental stress can already exist during pregnancy, it remains unclear whether there are also certain factors during pregnancy that could protect against later parental stress. These factors could potentially promote (long-term) maternal well-being and child-related outcomes, especially considering the evidence that mothers with elevated levels of parental stress, and their child, are at an increased risk for negative outcomes. One factor that may positively influence maternal well-being during this vulnerable period is mindfulness. Mindfulness can be defined as one's full attention to experiences in a presentcentered, non-judging, and accepting manner (Brown and Ryan 2003; Kabat-Zinn 1990). Mindfulness can be conceptualized both as a trait (one's predisposition to be mindful, which is stable over time) and as a state (which is temporary and practiced during mindfulness meditations) (Kiken et al. 2015). Frequent meditation practice, leading to a more frequent state of mindfulness, may in the long run, increases trait mindfulness (Kiken et al. 2015). Studies have shown that trait mindfulness is positively related to psychological health (Keng et al. 2011), such as empathy, self-esteem, mental health, and emotion regulation (Baer et al. 2006; Brown and Ryan 2003; Dekeyser et al. 2008). Maternal trait mindfulness during pregnancy has also been shown to be negatively associated with concurrent psychological distress (Truijens et al. 2016; van den Heuvel et al. 2015) and found to be predictive of subsequent psychological distress (Braeken et al. 2017) and prenatal and postpartum bonding to the infant (Cohen 2010).

In a study by Gouveia et al. (2016), it was shown that trait mindfulness in parents was negatively associated with parental stress. This study drew upon the work of Duncan et al. (2009), who suggested that when mindfulness is applied to the social context of parenting and the parent-child relationship, it positively influences parenting qualities. Duncan et al. (2009) referred to these parental qualities that emerge from applying mindfulness in the parenting and the parent-child relationship as "mindful parenting." In this model of mindful parenting, it was assumed that mindfulness in parenting could be stemming either from a natural capacity or from an acquired capacity through the practice of mindfulness meditation. Duncan et al. (2009) described several dimensions of mindful parenting, and its possible benefits for the parent, the child, and the parent-child relationship. These dimensions of mindful parenting were integrated in a questionnaire developed by Duncan (2007), measuring mindful parenting. The original and extended form of this questionnaire was used in the following two studies that examined the association between mindfulness, mindful parenting, and parental stress. In the study mentioned above, Gouveia et al. (2016) found that the association between mindfulness and parental stress was mediated by mindful parenting. A study by Parent et al. (2016) confirmed the association between parental trait mindfulness and mindful parenting in different stages of childhood (young childhood, middle childhood, and adolescence). Higher levels of prenatal mindfulness may also be predictive of higher levels of postpartum mindful parenting and lower levels of parental stress.

As far as we know, no quantitative studies confirmed this association between mindfulness during pregnancy and parental stress after birth. Furthermore, these studies did not assess different facets of mindfulness but unidimensional dispositional mindfulness. However, in a qualitative study on the effects of a mindfulness training for pregnant women, the 
women reported effects on their parenting qualities, such as improvement in co-regulation, emotion, and an improvement in the ability to reconnect after rupture (Krongold 2011).

This negative association between mindfulness and mindful parenting on one hand and parental stress on the other hand implies that if mindfulness and mindful parenting are increased in parents, parental stress will decrease. Several studies on the effectiveness of mindfulness and mindful parenting trainings for parents have confirmed this hypothesis for different populations of parents, such as in parents referred to a child and youth mental health care center (Bögels et al. 2014), parents of young children with developmental delay (Neece 2014), parents of children with autism spectrum disorder (Cachia et al. 2016), parents with parental stress who participated in a training in a non-clinical setting (Potharst et al. 2018a), and parents with a baby or toddler (Zeegers et al. 2019). In the aforementioned studies, different mindfulness trainings were offered to parents, with different contact hours. Either the mindfulness-based stress reduction (MBSR; Kabat-Zinn 1990) training was used (Neece 2014) or trainings that were adjusted for (the specific situation of the) parents. In these adjusted trainings, similar mindfulness meditations to those used in the MBSR were taught (e.g. Bögels et al. 2014; Zeegers et al. 2019). Bögels and Restifo (2013) hypothesized that mindfulness training decreases parental stress by diminishing automatic stress responses, eliciting a fight, flight or freeze response from parents to stressful parenting situations, and increases parents' capability to take conscious decisions in such situations. They also suggested that mindfulness training may decrease possible negative attentional bias, and promote a more open, accepting a way of looking at the child and at difficulties in the parent-child relationship, resulting in lower levels of parental stress.

The question is whether trait mindfulness during pregnancy may also be associated with future parental stress beyond the postpartum period (up to 12 months after delivery). There is some preliminary evidence on the positive effects of mindfulness training during pregnancy with regard to psychological distress in the postpartum period, but the measurements did not include parental stress specifically (Dunn et al. 2012; Duncan et al. 2017). In these studies, the trainings used were adjusted for pregnant women, and were either based on mindfulness-based cognitive therapy (MBCT, Segal et al. 2002) in the case of the study by Dunn et al. (2012), or based on MBSR and the mindfulness-based childbirth and parenting program (MBCP, Bardacke 2012), in the study by Duncan et al. (2017). The interventions had in common that mindfulness meditations as used in the MBSR were used, and this was also the case for the MBCP training that was offered in a qualitative study on the effectiveness of a mindfulness training during pregnancy (Malis et al. 2017). In this study, participating mothers were interviewed in the postpartum period. They reported the use of mindfulness abilities (e.g., acceptance and attention to the present moment) as a way of coping with and decreasing the experience of stress, including parenting stress (Malis et al. 2017).

Although no evidence is available on the more long-term positive effect of mindfulness during pregnancy on parental stress, the effects in the prenatal and postpartum period may be meaningful because of the great importance of this time of transition, as the basis for the experience of motherhood and the parent-child relationship. A study on parental stress in women with postpartum depression also showed that parental stress persisted during the first year after birth, even though levels of depression decreased (Milgrom and McCloud 1996). In a study by Dipietro et al. (2008), a high stability of psychological distress over time was found, from the prenatal period, to 6 weeks postpartum, to toddlerhood ( 2 years postpartum). In a longitudinal study assessing parental stress and child temperament, maternal stress at 6 months predicted both maternal stress and child temperamental problems at the age of 5 (Pesonen et al. 2008). A study by Neece et al. (2012) that followed children from toddlerhood until 9 years of age showed that parental stress influenced child behavior problems, and vice versa, resulting in a mutually escalating (or deescalating) effect over time. These studies showed that parental stress tends to be stable over time and underline the importance of the prevention of high levels of parental stress.

Toddlerhood is a time associated with specific challenges for parents that can be associated with parental stress (Kwon et al. 2013), corresponding to toddlers having a high need for autonomy and independence on the one hand, and a high need for support in regulating his behavior and emotions on the other hand (Bechtel-Kuehne et al. 2016; NICHD Early Child Care Research Network 2004). Crnic and Booth (1991) studied parents' daily hassles (which was operationalized as challenging child behavior and tasks associated with parenting) in parents with children aged 9 to 12 months, 18 to 24 months, and 30 to 36 months, and found that this form of parental stress increased over time. A qualitative study by Kwon et al. (2013) found that behavioral and developmental characteristics of toddlers (e.g., stubbornness, aggressiveness, independence, and limited emotion regulation), as well as being unable to communicate efficiently with a toddler, were challenging and stressful aspects for parents of toddlers.

It is important to investigate whether the prenatal factor mindfulness is associated with later parental stress and may therefore be related to a healthy parenting environment, a good foundation for the parent-child relationship, and the wellbeing of the next generation. In the current study, three distinct factors of mindfulness during pregnancy as predictors of parental stress are studied: acting with awareness, nonjudging, and non-reacting. These three facets are selected because of their most consistent associations with psychological distress-related phenomena (Baer et al. 2006; Bohlmeijer et al. 2011). Based on the previous literature, we hypothesize that 
the mindfulness facets, acting with awareness, non-judging, and non-reacting, during pregnancy will be negatively associated with parental stress, after adjusting for effects of prenatal and maternal distress and child behavior problems.

\section{Method}

\section{Participants}

In the current study, 166 women were eligible for inclusion. This is a subsample of women who completed the Holistic Approach to Pregnancy and the first Postpartum Year (HAPPY) study, a large longitudinal prospective cohort study that followed women during pregnancy until 12 months postpartum $(N=2269)$. In total, a subsample of $984(43 \%)$ participants who were included in the HAPPY cohort between March 2013 and December 2013 were sent the mindfulness questionnaire at 22 weeks of pregnancy, of which 911 (93\%) completed the questionnaire. Two and/or 3 years after giving birth, a subsample of participants of the HAPPY cohort were invited to participate in a follow-up study. Of the 911 women who completed the mindfulness questionnaire, 266 were approached to participate. Of these women, 170 (64\%) agreed to participate 3 years postpartum, and $166(98 \%)$ completed questionnaires on parental stress. This resulted in a subgroup of 166 women that were sent and completed both the mindfulness and parental stress questionnaires, and were consequently eligible for inclusion in the current study.

The aim of completing the assessment during pregnancy was at 22 weeks of pregnancy, with the window for completing the pregnancy assessment set at 18 to 26 weeks of pregnancy (this assessment is referred to as 22 weeks of pregnancy in the remainder of the manuscript). This criterion was not met by one participant. This resulted in a final sample of 165 women to be included for analysis. Characteristics of the participating women are shown in Table 1. According to a power analysis conducted in $\mathrm{G}^{*}$ power for multiple regression analyses establishing the power of a predictor with nine other predictors for a medium effect size, $\alpha$ of 0.05 and a power of 0.95 , the sample size required was 89 .

\section{Procedure}

Details regarding the design of the HAPPY study are reported elsewhere (Truijens et al. 2014). Seventeen community midwifery practices in the south of the Netherlands participated and approached Dutch-speaking Caucasian women who were eligible to participate at their first prenatal appointment. Inclusion took place from January 2013 to September 2014. Exclusion criteria were multiple pregnancy, a severe psychiatric disorder (e.g., schizophrenia, borderline personality disorder, and bipolar disorder), a documented history of chronic
Table 1 Characteristics of the participating women $(\mathrm{N}=165)$

\begin{tabular}{lllll}
\hline & $N$ & $\%$ & Mean (SD) & Range \\
\hline Maternal characteristics & & & $31.0(3.6)$ & $21-40$ \\
Age & & & & \\
Level of education & & & & \\
Low & 6 & 3.7 & & \\
Medium & 33 & 20.1 & & \\
High & 125 & 76.2 & & \\
Paid job & 151 & 91.5 & & \\
Living with partner & 165 & 100 & & \\
Child characteristics & & & & \\
Gender & & & & \\
Girl & 83 & 50.3 & & \\
Boy & 81 & 49.1 & & \\
Birth weight (grams) & & & $3478.6(434.1)$ & \\
Gestational age (weeks) & & & $39.8(1.28)$ \\
Number of children living in the household & \\
One & 25 & 15.2 & \\
Two & 106 & 64.2 & \\
Three or more & 34 & 20.6 & \\
\hline
\end{tabular}

$S D$ standard deviation, level of education, low primary education or secondary pre-vocational education, medium secondary education or vocational education, high Bachelor or Master's degree

disease (e.g., diabetes and thyroid dysfunction), and very preterm childbirth (gestational age $<32$ weeks).

The current cohort (HAPPY-follow) is a subsample of the HAPPY cohort. As part of the inclusion criteria, three different groups of women were selected to be included in this subsample. First, this concerned a group of women with high scores of depressive symptoms during pregnancy or postpartum $(N=68)$. A second group consisted of women with suboptimal thyroid function or thyroid dysfunction during early pregnancy $(N=22)$. Finally, the third and largest group consisted of healthy control women with thyroid function within trimester specific reference limits and no elevated depressive symptoms $(N=$ $83)$. These groups were selected for purposes not relevant to the current study. Data were collected from December 2016 to June 2018. Participants were asked to complete online and/or written questionnaires at various times from pregnancy onwards.

The HAPPY study was approved by the Psychology Ethics Committee of the Tilburg University (protocol number EV2012.25) and reviewed by the Medical Ethics Committee of the Máxima Medical Centre in Veldhoven, the Netherlands. The HAPPY follow study was approved by the Medical Ethics Committee of the Máxima Medical Centre (protocol number NL54558.015.15). Informed consent was obtained from all individual participants included in the study. 


\section{Measures}

\section{Demographics}

Women provided demographic information at 12 weeks of pregnancy, such as age and educational level (low = primary education or secondary pre-vocational education, medium = secondary education or vocational education, and high $=$ Bachelor or Master's degree). Obstetric features regarding gestational age (weeks), birthweight (grams), and gender of the child (boy/girl) were collected. Three years after childbirth, women completed information about living with a partner $(\mathrm{y} / \mathrm{n})$, having a paid job $(\mathrm{y} / \mathrm{n})$, and the number of children living in the household.

\section{Mindfulness During Pregnancy}

At 22 weeks of pregnancy, mindfulness was assessed using the Dutch version of the 12-item Three Facet Mindfulness Questionnaire-Short Form (TFMQ-SF), which has been validated in pregnant samples (Truijens et al. 2016). The TFMQSF was established based on the short form version of the Five Facet Mindfulness Questionnaire (FFMQ, Baer et al. 2006; Bohlmeijer et al. 2011). The scale consists of three subscales, each assessing a different facet of mindfulness, namely, (1) acting with awareness, (2) non-judging, and (3) non-reacting. For each item, women were asked to indicate how this applied to them during their pregnancy, on a 5-point Likert scale $(1=$ never or very rarely true to $5=$ very often or always true). The subscale scores on the TFMQ-SF were used, with higher scores indicating greater levels of mindfulness. Examples of items from each subscale respectively are (1) "it seems I am 'running on automatic' without awareness of what I am doing" (reversed scoring), (2) "I tell myself I should not be feeling the way I am feeling" (reversed scoring), and (3) "when I have distressing thoughts or images, I feel calm soon after." The TFMQ-SF has been shown to have adequate reliability and validity, with Cronbach's alphas of $0.87,0.84$, and 0.81 , respectively, in a pregnant sample (Truijens et al. 2016). The Cronbach's alphas in the current study, for each subscale, were $0.86,0.81$, and 0.72 , respectively.

\section{Prenatal Depressive Symptoms}

Depressive symptoms were assessed using the Dutch version of the 10-item Edinburgh (Postnatal) Depression Scale (EDS, Cox et al. 1987) at 22 weeks of pregnancy. The EDS has been validated for use in non-postpartum women (Cox et al. 1996) and for use in a Dutch population, during pregnancy (Bergink et al. 2011) and postpartum (Pop et al. 1992). Items were completed on a 4-point Likert scale, with total scores ranging from 0 to 30. Higher scores indicate more symptoms of depression. The EDS is a reliable instrument for use during pregnancy, with a Cronbach's alpha of 0.84 in the second trimester of pregnancy (Bergink et al. 2011). The Cronbach's alpha of the EDS in the current sample was also 0.84 .

\section{Prenatal Distress}

At 22 weeks of pregnancy, prenatal distress was assessed using the 11-item negative affect (NA) subscale of the Tilburg Pregnancy Distress Scale (TPDS, Pop et al. 2011). The scale consists of items regarding worries about pregnancy, delivery, and the postpartum period. Examples of items are "the delivery is troubling me" and "I worry about the pregnancy." Items were completed on a 4-point Likert scale $(0=$ very often to $3=$ rarely/never), with total scores ranging from 0 to 33 . Higher scores indicate more pregnancy-specific distress. The TPDS and its subscales show good validity and reliability (Boekhorst et al. 2020; Pop et al. 2011). In a study investigating the psychometric properties of the TPDS, the TPDS-NA showed good internal consistency, with a Cronbach's alpha of 0.78 in the second trimester of pregnancy (Boekhorst et al. 2020). In the current sample, the Cronbach's alpha was 0.76 .

\section{Parental Stress}

Parental stress was assessed using the Dutch version of the 34item Parenting Stress Questionnaire (PSQ, Vermulst et al. 2012). The PSQ was established based on the Parenting Stress Index (Abidin 1983) and can be completed by parents of children between the ages of 0 and 18. In the current study, mothers completed the questionnaire when their child was 3 years of age. Three subscales of the PSQ were used, each assessing a different component of parental stress: (1) parentchild relationship problems (6 items), (2) parenting problems (6 items), and (3) parental role restriction ( 7 items). The parent-child relationship subscale refers to the positive thoughts and feelings a parent has towards their child. With the parenting problems subscale, the perceived competence in the parenting role is referred to, and addresses whether a parent can control, correct and guide their child when needed. Role restriction refers to perceiving restrictions in areas other than child raising, due to the child (Vermulst et al. 2012). Examples of items from each subscale respectively are (1) "I feel cheerful when my child is with me," (2) "I can calm my child down when he/she gets angry," and (3) "raising my child leaves me with too little personal time." Mothers were asked to complete questions regarding interactions and experiences with their child on a 4-point Likert scale $(1=$ not true, $2=$ somewhat true, $3=$ quite true, $4=$ very true). Higher scores indicate greater levels of parental stress. For parents of children aged zero to three, scores $\geq 11$ on the parent-child relationship problems subscale, $\geq 15$ on the parenting problems 
subscale, and $\geq 14$ on the parental role restriction subscale, are considered to be cut-off scores corresponding to moderate to severe problems (Vermulst et al. 2012). The PSQ is a valid and reliable instrument (Veerman et al. 2014; Vermulst et al. 2012), with Cronbach's alphas of $0.84,0.83$, and 0.79 , for the subscales, respectively (Veerman et al. 2014). In the current study, the Cronbach's alphas for the subscales were 0.85 , 0.86 , and 0.82 , respectively.

\section{Maternal Symptoms of Anxiety and Depression}

At 3 years after childbirth, symptoms of anxiety and depression over the past 2 weeks were assessed using the Patient Health Questionnaire-4 (PHQ-4, Kroenke et al. 2009). The four-item PHQ-4 consists of the General Anxiety Disorder-2 (GAD-2, Kroenke et al. 2007) and the Patient Health Questionaire-2 (PHQ-2, Kroenke et al. 2003). Items were rated on a 4-point Likert scale $(0=$ not at all, $1=$ several days, $2=$ more than half the days, $3=$ nearly every day), with higher scores indicating more symptoms of anxiety and depression. Examples of items are "not being able to stop or control worrying" (GAD-2) and "feeling down, depressed, or hopeless" (PHQ-2). The PHQ-4 has adequate reliability and validity (Kroenke et al. 2009; Löwe et al. 2010), and the Cronbach's alpha was reported as 0.82 in the general population (Löwe et al. 2010). In the current sample, the Cronbach's alpha was 0.86 .

\section{Child Behavior}

Child problem behavior over the past 2 months was assessed using the Child Behavior Checklist for Ages 1.5-5 (CBCL1 1 $12-5$, Achenbach and Rescorla 2000). The $\mathrm{CBCL}$ is a norm-referenced behavior rating scale. Mothers were asked to answer 99 items regarding their child's behavior on a 3-point Likert scale $(0=$ not at all, $1=$ sometimes, $2=$ often), with higher scores indicating more problem behavior. Total scores of the internalizing and externalizing problem behavior scales were used in the current study, which were then converted to a T-score. In turn, T-scores can be norm-referenced into ranges of concern, namely, "average," "subclinical," or "clinical." Examples of items, with regard to the child, are "gets too upset when separated from parents" (internalizing problem behavior) and "punishment does not change his/ her behavior" (externalizing problem behavior). The CBCL total scale has a Cronbach's alpha of 0.95 (Achenbach and Rescorla 2000). Furthermore, good internal consistency has also been reported for the CBCL $1 \frac{1}{2-5}$ across different societies, including the Netherlands (Rescorla et al. 2011).

\section{Data Analyses}

All variables had appropriate skewness and kurtosis values (between -2 and +2 ) for normal distribution (George and Mallery 2014), except for the TPDS-NA and the PHQ-4. These measures had high kurtosis and were therefore LOG transformed. Differences in characteristics between the current sample and the overall HAPPY sample were assessed using independent $t$ tests and chi-square tests. Pearson's $r$ correlations were calculated between the three facets of mindfulness during pregnancy (acting with awareness, non-judging, and non-reacting), the three components of parental stress (parent-child relationship problems, parenting problems, and role restriction), prenatal distress, prenatal depressive symptoms, maternal symptoms of depression and anxiety, and child problem behavior at age three.

Next, multiple linear regression was used, for which each component of parental stress was analyzed separately as outcome. The three facets of mindfulness during pregnancy were used as predictors. Possible covariates related to mindfulness and parental stress were considered. We controlled for maternal age, level of education, prenatal distress, prenatal depressive symptoms, number of children living in the household, maternal symptoms of depression and anxiety 3 years after childbirth, and child behavior problems at age three.

Finally, to assess differences in mindfulness during pregnancy between women who score above the (sub)-clinical cutoff on each subscale of parental stress and women who do not score above this cut-off, independent sample $t$ tests were used.

Analyses were completed in the Statistical Package for the Social Sciences (IBM SPSS version 23.0). Effect sizes were also calculated. For the $t$ test, the Cohen's $d$ was calculated $(0.20=$ small, $0.50=$ medium, and $0.80=$ large $)$, and for Pearson's correlations, the $r$ was considered $(0.10=$ small, $0.30=$ medium, and $0.50=$ large $)($ Cohen 1988).

\section{Results}

First, it was examined whether the current sample $(N=165)$ differed in characteristics compared with the sample who also completed mindfulness assessment during pregnancy but not the parental stress assessment at 3 years after childbirth $(N=$ 747). Results showed that the current sample was slightly older $(M=30.96)$ compared with the sample that was not assessed 3 years postpartum $(M=29.93, p=.001$, Cohen's $d=0.29)$, and varied regarding the gestational age $(M=$ 39.78 weeks and $M=39.54$, respectively, $p=0.046$, Cohen's $d=0.15$ ). The two groups additionally differed in terms of educational level, $p=.003$, phi coefficient $=0.11$ (small effect size), and the current sample being more highly educated. The current sample also scored slightly higher on the non-reacting subscale of the TFMQ-SF $(M=12.39)$ compared with the 
sample that was not assessed 3 years postpartum $(M=11.39$, $p=.005$, Cohen's $d=0.25$ ). However, effect sizes were small for all significant differences. The population samples were not different with regard to non-judging, acting with awareness, prenatal distress, prenatal depressive symptoms, and birthweight and sex of the child.

The Pearson $r$ correlations between all measures are shown in Table 2. Acting with awareness during pregnancy was significantly negatively associated with parent-child relationship problems $(r=-.299, p<.001)$, parenting problems $(r=-.318$, $p<.001)$, and role restriction $(r=-.221, p<.001) 3$ years after childbirth. Non-judging was significantly negatively associated with parenting problems $(r=-.165, p=.034)$. Nonreacting was marginally significantly negatively associated with parenting problems $(r=-.148, p=.058)$.

Multiple linear regression analyses were conducted to assess the association between the three facets of mindfulness during pregnancy and the three components of parental stress. Assumptions regarding multicollinearity were met in all regression models, with appropriate tolerance and variance inflation factor values.

With regard to parent-child relationship problems, the total model was significant, $F(11,151)=5.98, p<.001, \mathrm{R}^{2}=0.30$. After the adjustment for covariates (maternal age, maternal education, prenatal distress, prenatal depressive symptoms, symptoms of anxiety and depression 3 years after childbirth, number of children in the household, and child internalizing and externalizing problem behavior at age three), acting with awareness remained to be significantly and inversely associated with parent-child relationship problems $(\beta=-0.25$, $p=.003$ ), but non-judging and non-reacting were not (see Table 3).

In the multiple linear regression analysis predicting parenting problems, $40.2 \%$ of the variance was explained. The total model was significant, $F(11,151)=9.21, p<.001$. Acting with awareness $(\beta=-0.21, p=.006)$ and non-reacting $(\beta=$ $-0.20, p=.004$ ) were significantly and negatively associated with parenting problems, after adjusting for maternal age, maternal education, prenatal distress, prenatal depressive symptoms, symptoms of anxiety and depression 3 years after child birth, number of children in the household, and child internalizing and externalizing problem behavior at age three (see Table 4).

With regard to the multiple linear regression predicting role restriction, $17.4 \%$ of the variance was explained, and the total model was significant, $F(11,151)=2.88, p=.002$. Acting with awareness was a marginally significant predictor of role restriction ( $\beta=-0.16, p=.073)$. Non-judging and nonreacting were not significant predictors of role restriction reported by mothers 3 years after childbirth. In this model, child internalizing problem behavior, as well as maternal symptoms of anxiety and depression, was significantly associated with role restriction (see Table 5).
It was calculated how many participating women scored above the cut-off for moderate to severe problems, for each subscale of the PSQ. Of all participating women, 27 (16.4\%) reported moderate to severe parent-child relationship problems, $24(14.5 \%)$ reported moderate to severe parenting problems, and 33 (20\%) reported moderate to severe problems with regard to role restriction. An independent sample $t$ test showed a significant difference in acting with awareness during pregnancy between women with moderate to severe parent-child relationship problems $(M=12.48 ; \mathrm{SD}=2.99)$ and women without these problems $(M=14.86 ; \mathrm{SD}=2.91)$, $t(163)=3.86, p<.001$, Cohen's $d=0.80$, large effect size . With regard to parent-child relationship problems, no differences were found in non-judging $(p=0.99)$ and non-reacting $(p=0.93)$ during pregnancy. Furthermore, women without parental problems showed significantly higher levels of acting with awareness $(M=14.78 ; \mathrm{SD}=2.92)$ compared with women with moderate to severe parenting problems $(M=12.63$; $\mathrm{SD}=3.19), t(163)=3.30, p=0.001$, Cohen's $d=0.71$, medium to large effect size. No differences in non-judging ( $p=$ $0.19)$ and non-reacting $(p=0.11)$ were found between women who reported moderate to severe parenting problems and women who did not report these problems. With regard to role restriction, the difference in acting with awareness between women with moderate to severe role restriction problems $(\mathrm{M}=13.58 ; \mathrm{SD}=3.52)$ and women without these problems $(\mathrm{M}=14.69 ; \mathrm{SD}=2.89)$ approached significance at the $p<.05$ level $(t(163)=1.89, p=0.060$, Cohen's $d=0.35$, small effect size). With regard to role restriction, no significant differences were found in non-judging $(p=0.36)$ and non-reacting $(p=$ 0.89 ) between women with moderate to severe problems and women without problems. The differences in acting with awareness during pregnancy, per component of parental stress, are shown in Fig. 1.

\section{Discussion}

The aim of the current study was to examine the possible association of facets of mindfulness during pregnancy (acting with awareness, non-judging, and non-reacting) with three components of parental stress 3 years after childbirth (parent-child relationship problems, parenting problems and role restriction). We controlled for several possible covariates, including age, level of education, number of children in the household, prenatal distress, prenatal depressive symptoms, child behavior problems at age three, and maternal symptoms of depression and anxiety 3 years after childbirth. Results of the multiple linear regression analyses showed that prenatal acting with awareness was significantly and negatively associated with later parent-child relationship problems and parenting problems, and marginally so with role restriction. Nonreacting was a significant predictor for parenting problems 


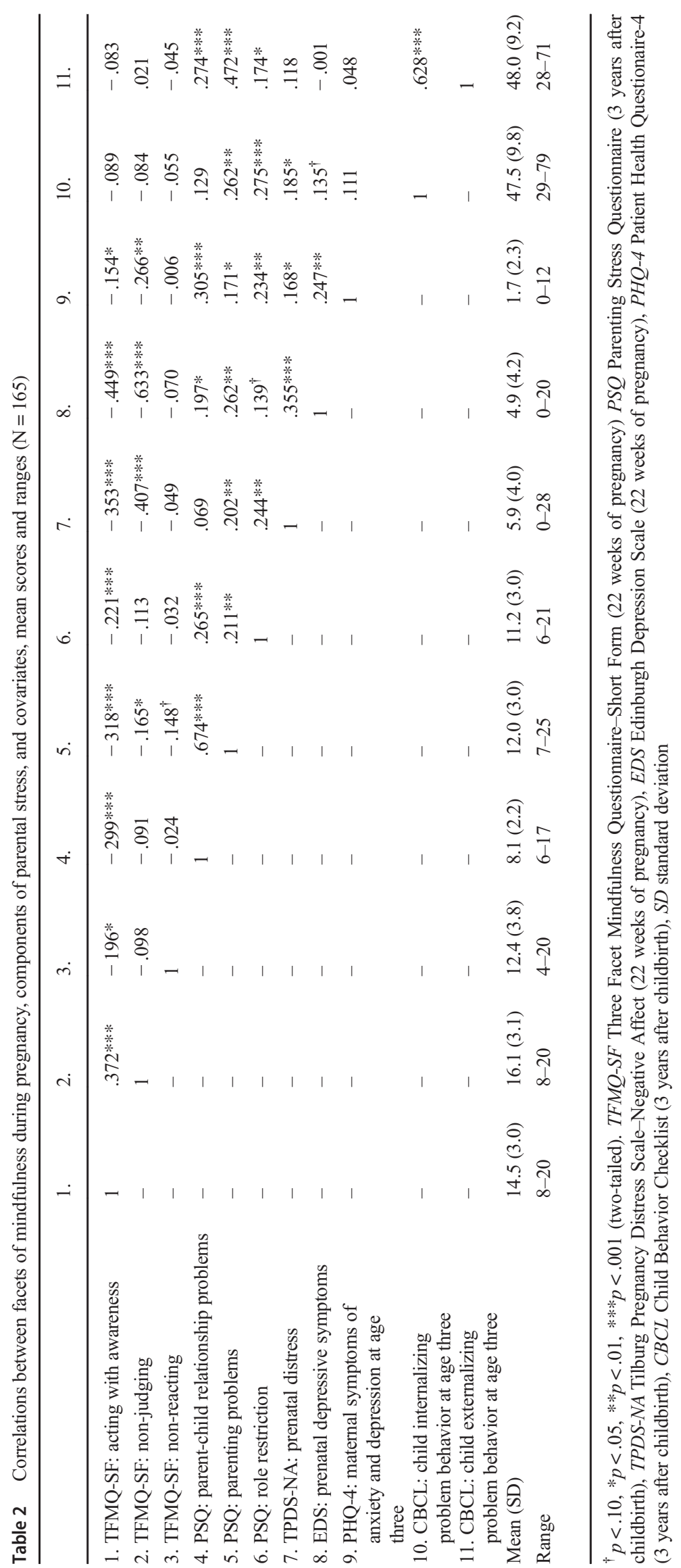


Table 3 Multiple regression predicting parent-child relationship problems $(\mathrm{N}=165)$

\begin{tabular}{lrrrl}
\hline & B (SE) & \multicolumn{1}{c}{$\beta$} & \multicolumn{1}{c}{$t$} & $95 \%$ CI \\
\hline TFMQ_SF: acting with awareness & $-0.18(0.06)$ & -0.25 & $-3.04^{* * *}$ & {$[-0.30,-0.06]$} \\
TFMQ_SF: non-judging & $0.06(0.07)$ & 0.08 & 0.90 & {$[-0.07,0.19]$} \\
TFMQ_SF: non-reacting & $-0.06(0.04)$ & -0.10 & -1.31 & {$[-0.14,0.03]$} \\
Maternal level of education & $1.02(0.30)$ & 0.24 & $3.45^{* *}$ & {$[0.44,1.61]$} \\
Maternal age & $0.01(0.05)$ & 0.01 & 0.18 & {$[-0.08,0.10]$} \\
Prenatal distress & $-0.56(0.66)$ & -0.07 & -0.85 & {$[-1.86,0.75]$} \\
Prenatal depressive symptoms & $0.05(0.05)$ & 0.10 & 1.04 & {$[-0.05,0.15]$} \\
Maternal symptoms of anxiety & $2.00(0.53)$ & 0.27 & $3.80^{* * *}$ & {$[0.96,3.04]$} \\
$\quad$ and depression at age three & $0.16(0.22)$ & 0.05 & 0.73 & {$[-0.28,0.60]$} \\
Number of children in household & $-0.02(0.02)$ & -0.10 & -1.06 & {$[0.06,0.02]$} \\
Child internalizing problem behavior & $0.08(0.02)$ & 0.33 & $3.65^{* * *}$ & {$[0.04,0.12]$} \\
Child externalizing problem behavior & &
\end{tabular}

$* * p<.01, * * * p<.001 ; B$ unstandardized regression coefficient, $S E$ standard error, $\beta$ standardized regression coefficient, $C I$ confidence interval, TFMQ-SF Three Facet Mindfulness Questionnaire-Short Form only, also showing a negative association. With regard to nonjudging, no significant associations were found.

The findings of the current study support the idea that the negative association between mindfulness and stress (Creswell and Lindsay 2014) is also applicable for parental stress in parents (Bögels et al. 2010; Duncan et al. 2009).

In the current study, three distinct factors of mindfulness during pregnancy as predictors of parental stress were studied: acting with awareness, non-judging, and nonreacting. These three facets were selected because of their most consistent associations with psychological distressrelated phenomena (Baer et al. 2006; Bohlmeijer, 2011). Of these three facets, acting with awareness (at 22 weeks of pregnancy) was the most strongly associated with parental stress (3 years postpartum) in the current study, even after adjusting for prenatal and maternal distress (at 22 weeks of pregnancy). A previous study also found a negative association between mindfulness and parental stress (Gouveia et al. 2016), in which mindfulness was assessed using the Mindful Attention and Awareness Scale (MAAS) (Brown and Ryan 2003). The focus of the MAAS is one's attention and awareness to occurrences in the present moment (Brown and Ryan 2003), and therefore overlaps with the concept assessed with the acting with awareness facet of the FFMQ. Furthermore, five out of eight items of the acting with awareness subscale of the FFMQ are derived from the MAAS (Baer et al. 2006). The findings from the study by Gouveia et al. (2016) therefore seem to implicate that acting with awareness is an important aspect of mindfulness that is negatively associated with parental stress.
Table 4 Multiple regression predicting parenting problems $(N=165)$

\begin{tabular}{lcccc}
\hline & B (SE) & $\beta$ & $t$ & $95 \%$ CI \\
\hline TFMQ_SF: acting with awareness & $-0.21(0.08)$ & -0.21 & $-2.78^{* * *}$ & {$[-0.36,-0.06]$} \\
TFMQ_SF: non-judging & $-0.03(0.09)$ & -0.03 & -0.36 & {$[-0.20,0.14]$} \\
TFMQ_SF: non-reacting & $-0.16(0.05)$ & -0.20 & $-2.89^{* *}$ & {$[-0.27,-0.05]$} \\
Maternal level of education & $0.69(0.38)$ & 0.12 & $1.81^{\dagger}$ & {$[-0.06,1.44]$} \\
Maternal age & $0.10(0.06)$ & 0.12 & $1.75^{\dagger}$ & {$[-0.01,0.22]$} \\
Prenatal distress & $0.62(0.85)$ & 0.06 & 0.74 & {$[-1.05,2.29]$} \\
Prenatal depressive symptoms & $0.09(0.06)$ & 0.12 & 1.32 & {$[-0.04,0.21]$} \\
Maternal symptoms of anxiety & $0.67(0.68)$ & 0.07 & 0.99 & {$[-0.67,2.00]$} \\
$\quad$ and depression at age three & $0.40(0.28)$ & 0.09 & 1.40 & {$[-0.16,0.95]$} \\
Number of children in household & $-0.03(0.03)$ & -0.08 & -0.97 & {$[-0.08,0.03]$} \\
Child internalizing problem behavior & $0.16(0.03)$ & 0.49 & $5.86^{* * *}$ & {$[0.11,0.22]$} \\
Child externalizing problem behavior & & & & \\
\hline
\end{tabular}

${ }^{\dagger} p<.10, * * p<.01, * * * p<.001 ; B$ unstandardized regression coefficient, $S E$ standard error, $\beta$ standardized regression coefficient, $C I$ confidence interval, TFMQ-SF Three Facet Mindfulness Questionnaire - Short Form 
Table 5 Multiple regression predicting role restriction $(N=$ 165)

\begin{tabular}{lrrrr}
\hline & B (SE) & \multicolumn{1}{c}{$\beta$} & \multicolumn{1}{c}{$t$} & \multicolumn{1}{l}{$95 \%$ CI } \\
\hline TFMQ_SF: acting with awareness & $-0.16(0.09)$ & -0.16 & $-1.81^{\dagger}$ & {$[-0.33,0.02]$} \\
TFMQ_SF: non-judging & $0.04(0.10)$ & 0.04 & 0.41 & {$[-0.15,0.24]$} \\
TFMQ_SF: non-reacting & $-0.04(0.06)$ & -0.05 & -0.68 & {$[-0.17,0.08]$} \\
Maternal level of education & $0.25(0.44)$ & 0.04 & 0.56 & {$[-0.62,1.11]$} \\
Maternal age & $0.05(0.07)$ & 0.06 & 0.74 & {$[-0.08,0.19]$} \\
Prenatal distress & $1.75(0.97)$ & 0.16 & $1.79^{\dagger}$ & {$[-0.18,3.67]$} \\
Prenatal depressive symptoms & $-0.03(0.07)$ & -0.04 & -0.34 & {$[-0.17,0.12]$} \\
Maternal symptoms of anxiety & $1.74(0.78)$ & 0.18 & $2.23 *$ & {$[0.20,3.28]$} \\
$\quad$ and depression at age three & $-0.22(0.33)$ & -0.05 & -0.66 & {$[-0.86,0.43]$} \\
Number of children in household & $0.08(0.02)$ & 0.25 & $2.48^{*}$ & {$[0.02,0.14]$} \\
Child internalizing problem behavior & $-0.01(0.03)$ & -0.03 & -0.27 & {$[-0.07,0.06]$} \\
Child externalizing problem behavior & &
\end{tabular}

${ }^{\dagger} p<.10, * p<.05 ; B$ unstandardized regression coefficient, $S E$ standard error, $\beta$ standardized regression coefficient, $C I$ confidence interval, TFMQ-SF Three Facet Mindfulness Questionnaire-Short Form
Acting with awareness also seems to be an important predictor for symptoms of stress and perceived stress in general, unrelated to parenting. A study among intensive care nurses found that, compared with non-judging and non-reacting, acting with awareness was most strongly negatively correlated to perceived stress ( $\mathrm{Lu}$ et al. 2019). Furthermore, a study by Yang et al. (2017) assessed perceived stress in mental health professionals, finding that acting with awareness was the strongest predictor for levels of stress and burnout symptoms, compared with the other facets of mindfulness. Brown et al. (2015) found acting with awareness to be directly associated with psychological health outcomes in college students, including levels of stress. Studies have also found that acting with awareness was the most constant facet of mindfulness that moderated the association between perceived stress and depression (Bränström et al. 2011; Lu et al. 2019).

This relative importance of acting with awareness is, however, not consistent across studies as non-judging and nonreacting have also been found to be substantially related to lower symptoms of psychological distress (Baer et al. 2006; Bohlmeijer et al. 2011; De Bruin et al. 2012). The reason why non-judging and non-reacting showed weaker associations
Fig. 1 Differences in mean scores in prenatal acting with awareness between mothers without problems and mothers with moderate/severe problems regarding the parent-child relationship, parenting and role restriction at 3 years postpartum, defined based on the cut-off scores of the subscales of the Parenting Stress Questionnaire $(N=165)$

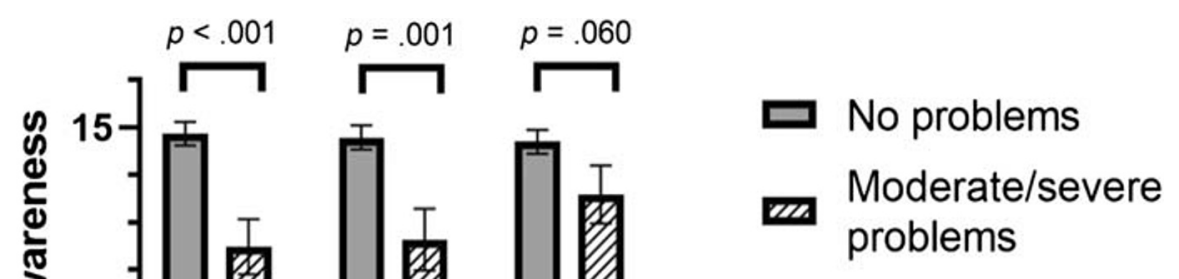


with parental distress is difficult to find. It may be speculated that the behavioral component of acting with awareness may be the factor making the difference. While non-judging and non-reacting are purely mental phenomena, acting with awareness implies a behavioral component reflecting attention to what is being done in the present moment. This facet may be particularly important in phenomena also having a strong behavioral component, such as interacting with one's child.

We found that acting with awareness at 22 weeks of pregnancy was a significant predictor of parent-child relationship problems 3 years postpartum. Seemingly, being attentive to the child and to the signals that the child gives is a requirement for the mother to respond appropriately to the needs of their child (Duncan et al. 2009). Ainsworth defined this appropriate responsiveness as maternal sensitivity (Ainsworth et al. 1974). The hypothesized association between sensitivity and mindfulness (specifically acting with awareness) has not been shown in previous research yet, but two studies did show an improvement of maternal sensitivity (Potharst et al. 2018b), and other aspects of adjustment and communication between mother and child (Zeegers et al. 2019) after the mothers had completed a mindfulness training for mothers with a baby or toddler. Improved sensitivity may therefore be an important mechanism explaining the relationship between acting with awareness during pregnancy and improved parent-child relationship.

Results of the current study showed that both acting with awareness and non-reacting at 22 weeks of pregnancy were significant predictors of parenting problems 3 years postpartum. The items on the parenting problems subscale of the PSQ refer to the ability to regulate the child's behavior in a wellattuned manner (e.g., being controllable to calm the child when upset, being able to correct their child when necessary) (Vermulst et al. 2012). With regard to acting with awareness, the results could imply that maternal awareness during pregnancy might be associated with mindful parenting when raising a toddler. Mindful parenting includes being able to focus the attention on the child in the present moment, and consciously choose responses towards the child, keeping in mind both the needs and inner experiences of the child and the self, as opposed to reacting to the child on autopilot. In turn, mindful parenting potentially increases behaviors of positive parenting and decrease behaviors of negative parenting (Parent et al. 2016), resulting in fewer parenting problems.

In addition, greater levels of non-reacting at 22 weeks of pregnancy were significantly associated with fewer parenting problems 3 years postpartum. Non-reactivity refers to selfregulatory processes and is defined as not getting absorbed in personal thoughts and feelings, but being able to let them come and go (Baer et al. 2006; Bohlmeijer et al. 2011). Nonreactivity is a construct not only important in general mindfulness, but also as a component of mindful parenting specifically (Duncan et al. 2009). Mindful parenting is hypothesized to lead to improved self-regulatory processes, for example, when a parent is able to notice their feelings and impulses, such as anger and screaming angrily, after which the parent can take a pause and choose a more appropriate response instead of acting out the impulse (Duncan et al. 2009; Bögels et al. 2010). A study on the Interpersonal Mindfulness in Parenting Scale (IM-P) showed that the emotional non-reactivity in parenting subscale of the IM-P was significantly positively correlated to the non-reacting subscale of the FFMQ and showed a significant negative correlation with an over-reactive parenting discipline (De Bruin et al. 2014). With regard to the results from the current study, this could imply that mothers who reported more non-reacting in pregnancy are, in turn, less reactive in parenting and parental discipline while raising their toddler, and thus show fewer parenting problems.

In the current study, it is shown that lower levels of mindfulness are associated with greater levels of parental stress. Earlier research also demonstrated that maternal internalizing problems (e.g., symptoms of depression and anxiety) could increase the risk for parental stress (Huizink et al. 2017; Misri et al. 2010). A study by Leigh and Milgrom (2008) showed a bidirectional process, in which not only maternal internalizing symptoms are a risk factor for parental stress, but parental stress is also a risk factor for maternal internalizing problems. Previous research has also shown that low levels of mindfulness are often associated with internalizing problems, such as symptoms of depression, stress, and anxiety (Tomlinson et al. 2018). The combination of these findings could indicate that especially women with internalizing problems may benefit from mindfulness-based interventions. Moreover, because parental stress is a risk factor for problems in the parent-child interaction as well as child behavioral outcomes (Barroso et al. 2018; McMahon and Meins 2012), it seems especially important to intervene early. Mindfulnessbased interventions might therefore prevent or minimize a negative cycle of problems when they are started at the first signs of symptoms. Yet, more research is needed on the (causal) role of mindfulness in the transactional relationship between parental stress and maternal symptomatology, and parental stress and child developmental outcomes.

\section{Limitations and Future Research}

A major strength is the longitudinal design of the current study, in which women were followed from pregnancy up until 3 years after childbirth, allowing us to study maternal functioning throughout this period of time. Another strength of our study is that different facets of mindfulness were assessed. Our study also has several limitations. A limitation is that the women in the current study population were more highly educated compared with the general population in the Netherlands (Statistics Netherlands 2019), and therefore 
limiting generalizability to the general population. A possible explanation of this difference could be that the current study took place in the south of the Netherlands, which is a more highly educated population compared with the general population of the Netherlands. This region was named "smartest area of the world" in 2011 by the international think-tank Intelligent Community Forum (ICF) in New York. Another limitation is that mindfulness and parental stress was only assessed in mothers and not fathers in the current study. Future studies should also take paternal mindfulness into consideration as previous studies have shown that in the early parenthood, mothers and fathers' parenting experiences vary with regard to the different components of parental stress (e.g., Hildingsson and Thomas 2014; Widarsson et al. 2013).

Another limitation of the current study is that the measures used in the current study were self-report (e.g., symptoms of distress, parental stress, child behavior). Furthermore, in the current study, a negative bivariate correlation between the non-reacting and the acting with awareness subscales was found. This is in line with the validation study of the TFMQ-SF, analyzed among the same study cohort of pregnant women as in the current study (Truijens et al. 2016). Lu et al. (2019) also found negative correlations between non-reactivity and acting with awareness in a sample of Chinese intensive care nurses. Yet, these correlations are different to findings with regard to studies on the FFMQ (Baer et al. 2006; De Bruin et al. 2012) and its short form (Bohlmeijer et al. 2011). A possible explanation of these differences, as suggested by Truijens et al. (2016), could be due to a different interpretation of the questions by pregnant women in comparison to other population samples. In the current study, participants were pregnant, and they were asked how each item of the TFMQ-SF applied to them in general during their pregnancy. Hence, they may interpret the non-reactivity items as letting go of thoughts and worries related to pregnancy and the unborn child. It could be that for pregnant women, it does not seem appropriate to let go of these thoughts or feelings, but rather to address them. This may be especially applicable when thoughts are related to the health of the fetus, by for example, discussing this with an obstetrician or midwife for assurance, before letting go of them.

Future research should further investigate the possible effects of mindfulness and mindfulness interventions during pregnancy, by more specifically addressing the parent-child relationship and attachment, as well as child developmental outcomes. Moreover, more research is needed on the implications of mindfulness during pregnancy on mindful parenting, and in turn, the effect on later parental stress across the different stages of child development. Furthermore, future research should examine the association between prenatal mindfulness on negative parenting behaviors and practices and its possible relationship with parental stress.
In conclusion, we found that aspects of mindfulness during pregnancy positively influence parental stress in mothers 3 years after childbirth, even after controlling for prenatal and maternal distress. Acting with awareness during pregnancy was found to be negatively associated with parent-child relationship problems and parenting problems 3 years later. Results also showed that non-reactivity during pregnancy predicted fewer parenting problems in mothers raising their toddler. These findings suggest that mindfulness during pregnancy may be a protective factor against later parental stress, which might benefit child outcomes.

Authors' Contributions MB contributed to data collection, data analysis, and wrote the paper. EP contributed to data analysis and wrote parts of the manuscript. $\mathrm{AB}$ collaborated in the writing and editing of the final manuscript. LH collaborated in the writing and editing of the final manuscript. VB collaborated in the writing and editing of the final manuscript. VP acquired funding, designed the study, monitored the process of data collection, collaborated in the writing, and editing of the manuscript. IN monitored to process of data analyses and writing of the study and collaborated in the writing and editing of the manuscript. All authors approved the final version of the manuscript.

Funding Information This study was funded by Stichting de Weijerhorst.

\section{Compliance with Ethical Standards}

Conflict of Interest The authors declare that they have no conflict of interest.

Ethical Approval All procedures performed in studies involving human participants were in accordance with the ethical standards of the institutional and/or national research committee (Psychology Ethics Committee at Tilburg University protocol number EV-2012.25 and the Medical Ethics Committee at the Máxima Medical Centre protocol number NL54558.015.15) and with the 1964 Helsinki declaration and its later amendments or comparable ethical standards.

Statement of Informed Consent Informed consent was obtained from all individual participants included in the study.

Open Access This article is licensed under a Creative Commons Attribution 4.0 International License, which permits use, sharing, adaptation, distribution and reproduction in any medium or format, as long as you give appropriate credit to the original author(s) and the source, provide a link to the Creative Commons licence, and indicate if changes were made. The images or other third party material in this article are included in the article's Creative Commons licence, unless indicated otherwise in a credit line to the material. If material is not included in the article's Creative Commons licence and your intended use is not permitted by statutory regulation or exceeds the permitted use, you will need to obtain permission directly from the copyright holder. To view a copy of this licence, visit http://creativecommons.org/licenses/by/4.0/.

\section{References}

Abidin, R. R. (1983). Parenting stress index manual. Charlottesville: Pediatric Psychology Press. 
Abidin, R. R. (1992). The determinants of parenting behavior. Journal of Clinical Child Psychology, 21(4), 407-412. https://doi.org/10.1207/ s15374424jccp2104 12.

Achenbach, T., \& Rescorla, L. (2000). Manual for the ASEBA preschool forms and profiles. Burlington: University of Vermont, Research Center for Children, Youth and Families.

Ainsworth, M. D. S., Bell, S. M., \& Stayton, D. J. (1974). Infant-mother attachment and social development: 'Socialization' as a product of reciprocal responsiveness to signals. In M. P. M. Richards (Ed.), The introduction of the child into a social world (pp. 99-135). London: Cambridge University Press.

Anthony, L. G., Anthony, B. J., Glanville, D. N., Naiman, D. Q., Waanders, C., \& Shaffer, S. (2005). The relationships between parenting stress, parenting behaviour and preschoolers' social competence and behaviour problems in the classroom. Infant and Child Development, 14, 133-154. https://doi.org/10.1002/icd.385.

Baer, R. A., Smith, G. T., Hopkins, J., Krietemeyer, J., \& Toney, L. (2006). Using self-report assessment methods to explore facets of mindfulness. Assessment, 13(1), 27-45. https://doi.org/10.1177/ 1073191105283504.

Bardacke, N. (2012). Mindful birthing: Training the mind, body, and heart for childbirth and beyond. New York: Haper One.

Barroso, N. E., Mendez, L., Graziano, P. A., \& Bagner, D. M. (2018). Parenting stress through the lens of different clinical groups: A systematic review \& meta-analysis. Journal of Abnormal Child Psychology, 46(3), 449-461. https://doi.org/10.1007/s10802-0170313-6.

Bechtel-Kuehne, S., Strodthoff, A., \& Pauen, S. (2016). Co- and selfregulation in the caregiver-child dyad: Parental expectations, children's compliance, and parental practices during early years. Journal of Self-Regulation and Regulation, 2, 33-56. https://doi. org/10.11588/josar.2016.2.34352.

Bergink, V., Kooistra, L., Lambregtse - van den Berg, M. P., Wijnen, H., Bunevicius, R., Van Baar, A., \& Pop, V. (2011). Validation of the Edinburgh depression scale during pregnancy. Journal of Psychosomatic Research, 70(4), 385-389. https://doi.org/10.1016/ j.jpsychores.2010.07.008.

Boekhorst, M. G. B. M., Beerthuizen, A., van Son, M., Bergink, V., \& Pop, V. J. M. (2020). Psychometric aspects of the Tilburg pregnancy distress scale: Data from the HAPPY study. Archives of Women's Mental Health, 23(2), 215-219. https://doi.org/10.1007/s00737019-00974-4 .

Bögels, S., \& Restifo, K. (2013). Mindful parenting: A guide for mental health practitioners. New York: Springer.

Bögels, S. M., Lehtonen, A., \& Restifo, K. (2010). Mindful parenting in mental health care. Mindfulness, 1(2), 107-120. https://doi.org/10. 1007/s12671-010-0014-5.

Bögels, S. M., Hellemans, J., van Deursen, S., Römer, M., \& van der Meulen, R. (2014). Mindful parenting in mental health care: Effects on parental and child psychopathology, parental stress, parenting, poparenting, and marital functioning. Mindfulness, 5(5), 536-551. https://doi.org/10.1007/s12671-013-0209-7.

Bohlmeijer, E., ten Klooster, P. M., Fledderus, M., Veehof, M., \& Baer, R. (2011). Psychometric properties of the five facet mindfulness questionnaire in depressed adults and development of a short form. Assessment, 18(3), 308-320. https://doi.org/10.1177/ 1073191111408231 .

Braeken, M. A. K. A., Jones, A., Otte, R. A., Nyklíček, I., \& Van den Bergh, B. R. H. (2017). Potential benefits of mindfulness during pregnancy on maternal autonomic nervous system function and infant development. Psychophysiology, 54(2), 279-288. https://doi. org/10.1111/psyp.12782.

Bränström, R., Duncan, L. G., \& Moskowitz, J. T. (2011). The association between dispositional mindfulness, psychological well-being, and perceived health in a Swedish population-based sample. British
Journal of Health Psychology, 16, 300-316. https://doi.org/10. 1348/135910710X501683.

Brown, K. W., \& Ryan, R. M. (2003). The benefits of being present: Mindfulness and its role in psychological well-being. Journal of Personality and Social Psychology, 84(4), 822-848. https://doi. org/10.1037/0022-3514.84.4.822.

Brown, D. B., Bravo, A. J., Roos, C. R., \& Pearson, M. R. (2015). Five facets of mindfulness and psychological health: Evaluating a psychological model of the mechanisms of mindfulness. Mindfulness, 6(5), 1021-1032. https://doi.org/10.1007/s12671-014-0349-4.

Cachia, R. L., Anderson, A., \& Moore, D. W. (2016). Mindfulness, stress and well-being in parents of children with autism spectrum disorder: A systematic review. Journal of Child and Family Studies, 25(1), 114. https://doi.org/10.1007/s10826-015-0193-8.

Cohen, J. (1988). Statistical power analysis for the behavioral sciences. Hillsdale: Lawrence Erlbaum Associates.

Cohen, J. S. (2010). Mindfulness and self-compassion in the transition to motherhood: A prospective study of postnatal mood and attachment (Doctoral dissertation). Retrieved August 18, 2019 from https:// pqdtopen.proquest.com/doc/858946838.html?FMT=AI . Accessed 18 Aug 2019.

Cox, J. L., Holden, J. M., \& Sagovsky, R. (1987). Detection of postnatal depression development of the 10-item Edinburgh postnatal depression scale. British Journal of Psychiatry, 150(6), 782-786. https:// doi.org/10.1192/bjp.150.6.782.

Cox, J. L., Chapman, G., Murray, D., \& Jones, P. (1996). Validation of the Edinburgh postnatal depression scale (EPDS) in non-postnatal women. Journal of Affective Disorders, 39(3), 185-189. https:// doi.org/10.1016/0165-0327(96)00008-0.

Creswell, J. D., \& Lindsay, E. K. (2014). How does mindfulness training affect health? A mindfulness stress buffering account. Current Directions in Psychological Science, 23(6), 401-407 10.1177\% 2F0963721414547415.

Crnic, K. A., \& Booth, C. L. (1991). Mothers' and fathers' perceptions of daily hassles of parenting across early childhood. Journal of Marriage and the Family, 53(4), 1042-1050. https://doi.org/10. $2307 / 353007$.

Crnic, K. A., Gaze, C., \& Hoffman, C. (2005). Cumulative parenting stress across the preschool period: Relations to maternal parenting and child behavior at age 5. Infant and Child Development, 14(2), 117-132. https://doi.org/10.1002/icd.384.

Cuijlits, I., van de Wetering, A. P., Endendijk, J. J., van Baar, A. L., Potharst, E. S., \& Pop, V. J. M. (2019). Risk and protective factors for pre-and postnatal bonding. Infant Mental Health Journal, 40(6), 768-785. https://doi.org/10.1002/imhj.21811.

De Bruin, E. I., Topper, M., Muskens, J. G. A. M., Bögels, S. M., \& Kamphuis, J. H. (2012). Psychometric properties of the five facets mindfulness questionnaire (FFMQ) in a meditating and a nonmeditating sample. Assessment, 19(2), 187-197. https://doi.org/10. 1177/1073191112446654.

De Bruin, E. I., Zijlstra, B. J. H., Geurtzen, N., Van Zundert, R. M. P., Van de Weijer-Bergsma, E., Hartman, E. E., Nieuwesteeg, A. M., Duncan, L. G., \& Bögels, S. M. (2014). Mindful parenting assessed further: Psychometric properties of the Dutch version of the interpersonal mindfulness in parenting scale (IM-P). Mindfulness, 5(2), 200-212. https://doi.org/10.1007/s12671-012-0168-4.

Deater-Deckard, K. (1998). Parenting stress and child adjustment: Some old hypotheses and new questions. Clinical Psychology: Science and Practice, 5(3), 314-332. https://doi.org/10.1111/j.1468-2850. 1998.tb00152.x.

Dekeyser, M., Raes, F., Leijssen, M., Leysen, S., \& Dewulf, D. (2008). Mindfulness skills and interpersonal behaviour. Personality and Individual Differences, 44(5), 1235-1245. https://doi.org/10.1016/ j.paid.2007.11.018.

Dennis, C., Falah-Hassani, K., \& Shiri, R. (2017). Prevalence of antenatal and postnatal anxiety: Systematic review and meta-analysis. The 
British Journal of Psychiatry, 210(5), 315-323. https://doi.org/10. 1192/bjp.bp.116.187179.

Dipietro, J. A., Costigan, K. A., \& Sipsma, H. L. (2008). Continuity in self-report measures of maternal anxiety, stress, and depressive symptoms from pregnancy through two years postpartum. Journal of Psychosomatic Obstetrics and Gynecology, 29(2), 115-124. https://doi.org/10.1080/01674820701701546.

Duncan, L. G. (2007). Assessment of mindful parenting among parents of early adolescents: Development and validation of the interpersonal mindfulness in parenting scale. (doctoral dissertation). State College: The Pennsylvania State University.

Duncan, L. G., Coatsworth, J. D., \& Greenberg, M. T. (2009). A model of mindful parenting: Implications for parent-child relationships and prevention research. Clinical Child and Family Psychology Review, 12(3), 255-270. https://doi.org/10.1007/s10567-009-0046-3.

Duncan, L. G., Cohn, M. A., Chao, M. T., Cook, J. G., Riccobono, J., \& Bardacke, N. (2017). Benefits of preparing for childbirth with mindfulness training: A randomized controlled trial with active comparison. BMC Pregnancy and Childbirth, 17(1), 140. https://doi.org/10. 1186/s12884-017-1319-3.

Dunn, C., Hanieh, E., Roberts, R., \& Powrie, R. (2012). Mindful pregnancy and childbirth: Effects of a mindfulness-based intervention on women's psychological distress and well-being in the perinatal period. Archives of Women's Mental Health, 15(2), 139-143. https:// doi.org/10.1007/s00737-012-0264-4.

George, D., \& Mallery, P. (2014). IBM SPSS statistics 21 step: A simple guide and reference (13th ed.). Boston: Pearson.

Glover, V., \& Capron, L. (2017). Prenatal parenting. Current Opinion in Psychology, 15, 66-70. https://doi.org/10.1016/j.copsyc.2017.02.007.

Gouveia, M. J., Carona, C., Canavarro, M. C., \& Moreira, H. (2016). Self-compassion and dispositional mindfulness are associated with parenting styles and parenting stress: The mediating role of mindful parenting. Mindfulness, 7(3), 700-712. https://doi.org/10.1007/ s12671-016-0507-y.

Haapsamo, H., Pollock-Wurman, R. A., Kuusikko-Gauffin, S., Ebeling, H., Larinen, K., Soini, H., \& Moilanen, I. (2013). Maternal stress and young children's behavioural development: A prospective pilot study from 8 to 36 months in a Finnish sample. Early Child Development and Care, 183(12), 1841-1852. https://doi.org/10. 1080/03004430.2012.756874.

Hildingsson, I., \& Thomas, J. (2014). Parental stress in mothers and fathers one year after birth. Journal of Reproductive and Infant Psychology, 32(1), 41-56. https://doi.org/10.1080/02646838.2013. 840882.

Huizink, A. C., Menting, B., De Moor, M. H. M., Verhage, M. L., Kunseler, F. C., Schuengel, C., \& Oosterman, M. (2017). From prenatal anxiety to parenting stress: A longitudinal study. Archives of Women's Mental Health, 20(5), 663-672. https://doi.org/10.1007/ s00737-017-0746-5.

Kabat-Zinn, J. (1990). Full catastrophe living: Using the wisdom of your body and mind to face stress, pain, and illness. New York: Delacorte.

Keng, S. L., Smoski, M. J., \& Robins, C. J. (2011). Effects of mindfulness on psychological health: A review of empirical studies. Clinical Psychology Review, 31(6), 1041-1056. https://doi.org/10.1016/j. cpr.2011.04.006.

Kerstis, B., Nohlert, E., Öhrvik, J., \& Widarsson, M. (2016). Association between depressive symptoms and parental stress among mothers and fathers in early parenthood: A Swedish cohort study. Upsala Journal of Medical Sciences, 121(1), 60-64. https://doi.org/10. 3109/03009734.2016.1143540.

Kiken, L. G., Garland, E. L., Bluth, K., Palsson, O. S., \& Gaylord, S. A. (2015). From a state to a trait: Trajectories of state mindfulness in meditation during intervention predict changes in trait mindfulness. Personality and Individual Differences, 81, 41-46. https://doi.org/ 10.1016/j.paid.2014.12.044.
Kroenke, K., Spitzer, R. L., \& Williams, J. B. (2003). The patient health questionnaire- 2: Validity of a two-item depression screener. Medical Care, 41(11), 1284-1292. https://doi.org/10.1097/01. MLR.0000093487.78664.3C.

Kroenke, K., Spitzer, R. L., Williams, J. B., Monahan, P. O., \& Löwe, B. (2007). Anxiety disorders in primary care: Prevalence, impairment, comorbidity, and detection. Annals of Internal Medicine, 146(5), 317-325. https://doi.org/10.7326/0003-4819-146-5-20070306000004.

Kroenke, K., Spitzer, R. L., Williams, J. B. W., \& Löwe, B. (2009). An ultra-brief screening scale for anxiety and depression: The PHQ 4 . Psychosomatics, 50(6), 613-621. https://doi.org/10.1016/S00333182(09)70864-3.

Krongold, K. S. (2011). Mindfulness-based prenatal care and postnatal mother-infant relationships (doctoral dissertation). San Francisco: California Institute of Integral Studies.

Kwon, K. A., Han, S., Jeon, H. J., \& Bingham, G. E. (2013). Mothers' and fathers' parenting challenges, strategies, and resources in toddlerhood. Early Child Development and Care, 183(3-4), 415-419. https://doi.org/10.1080/03004430.2012.711591.

Leigh, B., \& Milgrom, J. (2008). Risk factors for antenatal depression, postnatal depression and parenting stress. BMC Psychiatry. https:// doi.org/10.1186/1471-244X-8-24.

Löwe, B., Wahl, I., Rose, M., Spitzer, C., Glaesmer, H., Wingenfeld, K., Schneider, A., \& Brähler, E. (2010). A 4-item measure of depression and anxiety: Validation and standardization of the patient health Questionnaire-4 (PHQ-4) in the general population. Journal of Affective Disorders, 122, 86-95. https://doi.org/10.1016/j.jad.2009. 06.019 .

Lu, F., Xu, Y., Yu, Y., Peng, L., Wu, T., Wang, T., Liu, B., Xie, J., Xu, S., $\& \mathrm{Li}$, M. (2019). Moderating effect of mindfulness on the relationships between perceived stress and mental health outcomes among Chinese intensive care nurses. Frontiers in Psychiatry. https://doi. org/10.3389/fpsyt.2019.00260 .

Malis, F. R., Meyer, T., \& Gross, M. M. (2017). Effects of an antenatal mindfulness-based childbirth and parenting programme on the postpartum experiences of mothers: A qualitative interview study. $B M C$ Pregnancy and Childbirth, 17(1), 57. https://doi.org/10.1186/ s12884-017-1240-9.

Matthijssen, J. J. J. P., Koot, H. M., Verhulst, F. C., De Bruyn, E. E. J., \& Oud, J. H. L. (1998). The relationship between mutual family relations and child psychopathology. Journal of Child Psychology and Psychiatry, 39, 477-487. https://doi.org/10.1111/1469-7610.00344.

McMahon, C. A., \& Meins, E. (2012). Mind-mindedness, parenting stress, and emotional availability in mothers of preschoolers. Early Childhood Research Quarterly, 27(2), 245-252. https://doi.org/10. 1016/j.ecresq.2011.08.002.

Mihelic, M., Filus, A., \& Morawaska, A. (2016). Correlates of prenatal parenting expectations in new mothers: Is better self-efficacy a potential target for preventing postnatal adjustment difficulties? Prevention Science, 17(8), 949-959. https://doi.org/10.1007/ s11121-016-0682-z.

Milgrom, J., \& McCloud, P. (1996). Parenting stress and postnatal depression. Stress Medicine, 12(3), 177-186. https://doi.org/10.1002/ (SICI)1099-1700(199607)12:3\%3C177::AID-SMI699\%3E3.0.CO; 2-W.

Misri, S., Kendrick, K., Oberlander, T. F., Norris, S., Tomfohr, L., Zhang, H., \& Grunau, R. E. (2010). Antenatal depression and anxiety affect postpartum parenting stress: A longitudinal, prospective study. The Canadian Journal of Psychiatry, 55(4), 222-228. https://doi.org/10. 1177/070674371005500405.

Moe, V., Von Soest, T., Fredriksen, E., Olafsen, K. S., \& Smith, L. (2018). The multiple determinants of maternal parenting stress 12 months after birth: The contribution of antenatal attachment style, adverse childhood experiences, and infant temperament. Frontiers in Psychology. https://doi.org/10.3389/fpsyg.2018.01987. 
Neece, C. L. (2014). Mindfulness-based stress reduction for parents of young children with developmental delays: Implications for parental mental health and child behavior problems. Journal of Applied Research in Intellectual Disabilities, 27(2), 174-186. https://doi. org/10.1111/jar.12064.

Neece, C. L., Green, S. A., \& Baker, B. L. (2012). Parenting stress and child behavior problems: A transactional relationship across time. American Journal on Intellectual and Developmental Disabilities, 117(1), 48-66. https://doi.org/10.1352/1944-7558-117.1.48.

NICHD Early Child Care Research Network. (2004). Affect dysregulation in the mother-child relationship in the toddler years: Antecedents and consequences. Development and Psychopathology, 16, 43-68. https://doi.org/10.1017/ S0954579404044402.

Nyström, K., \& Öhrling, K. (2003). Parenthood experiences during the child's first year: Literature review. Journal of Advanced Nursing, 46(3), 319-330. https://doi.org/10.1111/j.1365-2648.2004.02991.x.

Östberg, M., Hagekull, B., \& Hagelin, E. (2007). Stability and prediction of parenting stress. Infant and Child Development, 16(2), 207-223. https://doi.org/10.1002/icd.516.

Parent, J., McKee, L. G., Rough, J. N., \& Forehand, R. (2016). The association of parent mindfulness with parenting and youth psychopathology across three developmental stages. Journal of Abnormal Child Psychology, 44(1), 191-202. https://doi.org/10.1007/s10802015-9978-x.

Pesonen, A. K., Räikkönen, K., Heinonen, K., Komsi, N., Järvenpää, A. L., \& Strandberg, T. (2008). A transactional model of temperamental development: Evidence of a relationship between child temperament and maternal stress over five years. Social Development, 17(2), 326340. https://doi.org/10.1111/j.1467-9507.2007.00427.x.

Pop, V. J., Komproe, I. H., \& Van Son, M. J. (1992). Characteristics of the Edinburgh post Natal depression scale in the Netherlands. Journal of Affective Disorders, 26(2), 105-110. https://doi.org/10.1016/01650327(92)90041-4.

Pop, V. J. M., Pommer, A. M., Pop-Purceleanu, M., Wijnen, H. A. A., Bergink, V., \& Pouwer, F. (2011). Development of the Tilburg pregnancy distress Scale: The TPDS. BMC Pregnancy and Childbirth. https://doi.org/10.1186/1471-2393-11-80.

Potharst, E. S., Baartmans, J. M., \& Bögels, S. M. (2018a). Mindful parenting training in a clinical versus non-clinical setting: An explorative study. Mindfulness. https://doi.org/10.1007/s12671-0181021-1.

Potharst, E. S., Zeegers, M., \& Bögels, S. M. (2018b). Mindful with your toddler group training: Feasibility, acceptability, and effects on subjective and objective measures. Mindfulness. https://doi.org/10. 1007/s12671-018-1073-2.

Rescorla, L. A., Achebach, T. M., Ivanova, M. Y., Harder, V. S., Otten, L., et al. (2011). International comparisons of behavioral and emotional problems in preschool children: parents' reports from 24 societies. Journal of Clinical Child and Adolescent Psychology, 40(3), 456467. https://doi.org/10.1080/15374416.2011.563472.

Saisto, T., Salmela-Aro, K., Nurmi, J., \& Halmesmäki, E. (2008). Longitudinal study on the predictors of parental stress in mothers and fathers of toddlers. Journal of Psychosomatic Obstetrics and Gynecology, 29(3), 213-222. https://doi.org/10.1080/ 01674820802000467.

Segal, Z. V., Williams, J. M. G., \& Teasdale, J. D. (2002). Mindfulnessbased cognitive therapy for depression: A new approach to preventing relapse. Guilford Press.
Statistics the Netherlands (2019). Population; educational level; gender, age and migration background (Bevolking; onderwijsniveau; geslacht, leeftijd en migratieachtergrond) https://statline.cbs.nl/ Statweb/publication/?DM $=$ SLNL \&PA $=82275$ NED $\& D 1=0 \& D 2=$ $1 \& \mathrm{D} 3=0,2-8 \& \mathrm{D} 4=0-1,3-4 \& \mathrm{D} 5=0,3,5-6,9-11,14,16-17 \& \mathrm{D} 6=$ $54,59,64,69,74,79-80 \& H D R=T, G 1, G 5, G 2 \& S T B=G 3, G 4 \& V W=T$ (accessed 16 May 2019).

Stern, D. N. (2018). The motherhood constellation: A unified view of parent-infant psychotherapy. Routledge.

Tomlinson, E. R., Yousaf, O., Vittersø, A. D., \& Jones, L. (2018). Dispositional mindfulness and psychological health: A systematic review. Mindfulness, 9(1), 23-43. https://doi.org/10.1007/s12671017-0762-6.

Truijens, S. E. M., Meems, M., Kuppens, S. M. I., Broeren, M. A. C., Nabbe, K. C. A. M., Wijnen, H. A., Oei, G., Van Son, M. J. M., \& Pop, V. J. M. (2014). The HAPPY study (holistic approach to pregnancy and the first postpartum year): Design of a large prospective cohort study. BMC Pregnancy and Childbirth. https://doi.org/10. 1186/1471-2393-14-312.

Truijens, S. E., Nyklíček, I., van Son, J., \& Pop, V. J. M. (2016). Validation of a short form three facet mindfulness questionnaire (TFMQ-SF) in pregnant women. Personality and Individual Differences, 93, 118-124. https://doi.org/10.1016/j.paid.2015.06. 037.

Van den Heuvel, M. I., Johannes, M. A., Henrichs, J., \& Van den Bergh, B. R. H. (2015). Maternal mindfulness during pregnancy and infant socio-emotional development and temperament: The mediating role of maternal anxiety. Early Human Development, 91(2), 103-108. https://doi.org/10.1016/j.earlhumdev.2014.12.003.

Veerman, J. W., Kroes, G., De Meyer, R. E., Nguyen, L. M., \& Vermulst, A. A. (2014). Opvoedingsbelasting in kaart gebracht. Een kennismaking met de opvoedingsbelastingvragenlijst (OBVL). JGZ Tijdschrift voor Jeugdgezondheidszorg, 46(3), 51-55. https:// doi.org/10.1007/s12452-014-0016-0.

Vermulst, A., Kroes, G., De Meyer, R., Nguyen, L., \& Veerman, J. W. (2012). Opvoedingsbelastingvragenlijst (OBVL). Handleiding. Nijmegen: Praktikon.

Widarsson, M., Engström, G., Rosenblad, A., Kerstis, B., Edlund, B., \& Lundberg, P. (2013). Parental stress in early parenthood among mothers and fathers in Sweden. Scandinavian Journal of Caring Sciences, 27(4), 839-847. https://doi.org/10.1111/j.1471-6712. 2012.01088.x.

Woody, C. A., Ferrari, A. J., Siskind, D. J., Whiteford, H. A., \& Harris, M. G. (2017). A systematic review and meta-regression of the prevalence and incidence of perinatal depression. Journal of Affective Disorders, 219, 86-92. https://doi.org/10.1016/j.jad.2017.05.003.

Yang, S., Meredith, P., \& Khan, A. (2017). Is mindfulness associated with stress and burnout among mental health professionals in Singapore? Psychology, Health \& Medicine, 22(6), 673-679. https://doi.org/10. 1080/13548506.2016.1220595.

Zeegers, M. A. J., Potharst, E. S., Veringa-Skiba, I. K., Aktar, E., Goris, M., Bögels, S. M., \& Colonnesi, C. (2019). Evaluating mindful with your baby/toddler: Observational changes in maternal sensitivity, acceptance, mind-mindedness, and dyadic synchrony. Frontiers in Psychology. https://doi.org/10.3389/fpsyg.2019.00753.

Publisher's Note Springer Nature remains neutral with regard to jurisdictional claims in published maps and institutional affiliations. 